\title{
Transmissão urbana de esquistossomose em crianças de um bairro de Salvador, Bahia
}

\author{
Urban transmission of schistosomiasis in children \\ from a neighborhood of Salvador, Bahia
}

\author{
Isabel C.S. Guimarães ${ }^{1}$ e José Tavares-Neto
}

\begin{abstract}
RESUM0
Na região do Subúrbio Ferroviário da Cidade do Salvador (Bahia, Brasil), foram relatados casos de esquistossomose, e isso motivou este estudo de prevalência em 268 escolares residentes em um dos seus bairros (São Bartolomeu). Em 30,2\% das crianças, o exame parasitológico (Kato-Katz) foi positivo, com carga parasitária entre 24 a 2.160 ovos de Schistosoma mansoni/g de fezes, predominando entre os meninos e nos maiores de 10 anos de idade. Nas crianças com residências mais próximas das coleções naturais de água, ocorreu maior freqüência de eliminadores de ovos e nela residiam os três únicos casos com a forma hepatosplênica da esquistossomose. Também, todas as 81 crianças com ovos de Schistosoma mansoni nas fezes nasceram e sempre residiram na área de estudo. Como nesse bairro há todos os elementos da cadeia epidemiológica do Schistosoma mansoni, são fortes as evidências da transmissão urbana dessa infecção.
\end{abstract}

Palavras-chaves: Esquistossomose mansônica. Transmissão urbana. Escolares. Salvador. Bahia.

\begin{abstract}
Tradicionalmente no Brasil, a esquistossomose mansônica é considerada como endemia rural, mas na atualidade há crescente número de casos notificados em cidades de maior porte $^{12}$. Em Belo Horizonte (MG), por exemplo, há focos naturais de infecção pelo Schistosoma mansoni com grande número de casos identificados nas últimas décadas ${ }^{14}$. Não obstante, a concentração de casos da doença em área urbana não necessariamente denuncia transmissão do Schistosoma mansoni, porque pode ser apenas o resultado da migração de população de região endêmica.
\end{abstract}

In the Subúrbio Ferroviário region of Salvador (Bahia, Brasil) occurrences of schistosomiasis were found. This fact motivated the investigation of the prevalence of the eggs in a group of 268 resident students in one of its neighborhoods ( São Bartolomeu). The parasitological test analyzed by the Kato-Katz methodology, showed positive results in $30.2 \%$ of the children, ranging from 24 to 2,160 eggs Schistosoma mansoni/gram of feces. Infection was predominant in boys and in those 10 years old and over. Observation revealed that infection predominated in children that live close to natural water bodies. This region was where the three registered cases of hepatosplenicos occurred. Moreover, all the 81 children infected with Schistosoma mansoni eggs were born and live in the study area. Since this neighborhood presents all the elements of the epidemiological chain of Schistosoma mansoni, the evidences strongly suggests the urban transmission of this infection.

Key-words: Schistosomiasis mansoni. Urban transmission. School children. Salvador. Bahia.

Mesmo não incluindo as áreas rurais, o levantamento de Pellon \& Teixeira ${ }^{16}$ em escolares das sedes de pequenas cidades, especialmente do nordeste brasileiro e do norte do Estado de Minas Gerais, foi o primeiro a evidenciar 0 impacto da esquistossomose em larga extensão territorial do Brasil. Quase 25 anos depois, a distribuição da esquistossomose no Brasil ficou ainda mais caracterizada com a publicação do levantamento de Freitas ${ }^{10}$. Nas últimas três décadas do século XX, também foi observada a progressiva redução da prevalência de esquistossomose em muitas localidades

1. Secretaria Municipal de Saúde, Salvador, BA. 2. Curso de Pós-graduação em Medicina e Saúde da Faculdade de Medicina da Bahia da Universidade Federal da Bahia, Salvador, BA.

Financiamento: Secretaria Municipal de Saúde de Salvador, FUNASA, CNPq, FAPESB, PET-Medicina.

Endereço para correspondência: Dra. Isabel C.S. Guimarães. Rua Arthur Fraga 276/203, Edifício Rio Moxotó, STIEP, 41770-030 Salvador, BA.

Tel: $55713341-0811$.

e-mail: jungbel@ig.com.br

Recebido para publicação em 6/6/2005

Aceito em 20/7/2006 
brasileiras e 0 crescente número de casos em cidades de maior porte ${ }^{2}$. Essas mudanças do padrão epidemiológico da esquistossomose foram em grande parte decorrente do significativo êxodo rural, a partir dos anos 60 , e também de técnicas diagnósticas mais eficientes ou mais aplicáveis em inquéritos populacionais; da introdução de novas drogas esquistossomicidas; e das melhorias nas condições do sistema de saneamento básico ${ }^{12}$.

№ Estado de Pernambuco, Barbosa e cols ${ }^{4}$ associaram a urbanização da esquistossomose como decorrência da migração de pessoas procedentes de áreas rurais ou de pequenas localidades na busca por trabalho nas cidades de maior porte, onde são freqüentemente marginalizadas do processo econômico, e muitas vêem a residir em áreas urbanas sem as mínimas condições básicas de saneamento. Com essa população migrante, aquelas áreas com cursos naturais de áqua, de características adequadas, e a presença de criadouros naturais de planorbídeos passaram a ser focos de transmissão da infecção pelo $S$. mansoni. Por isso, no Brasil muitos autores associam 0 fenômeno de urbanização da esquistossomose com componentes socioeconômicos e comportamentais ${ }^{3459}$.

No Estado da Bahia, em estudo baseado em dados secundários de quatro décadas, Barreto e cols ${ }^{7}$ encontraram prevalência média de 15,6\% no ano de 1950 e de 9,5\% em 1994 e, segundo os mesmos autores, a urbanização e a migração estão associadas a essa variação da prevalência, indicando também vinculação entre 0 processo da dinâmica populacional e a disseminação da doença nos diversos espaços que constituem 0 território baiano. Na Cidade do Salvador, capital do Estado, nos anos 50 e 60 do século passado eram conhecidos focos naturais de infecção pelo $S$. mansoni em áreas do perímetro urbano (Dique do Tororó) e em lagoas localizadas em bairros mais periféricos ( e.g., Placaford, Parque de Pituaçu, Jaguaribe e adjacências, entre outras) ${ }^{6}$.

A partir do ano 2001, na Cidade do Salvador foi maior 0 avanço do processo de municipalização dos serviços de assistência à saúde e por essa época passou a ser observado 0 crescente número de prescrições de oxamniquine ou praziquantel por parte de médicos de algumas equipes do Programa de Saúde da Família (PSF) do Subúrbio Ferroviário. As investigações epidemiológicas, subseqüentes, mostraram que àquelas prescrições tinham procedência de equipes localizadas especialmente na área do bairro de São Bartolomeu, onde há fontes de água naturais (lagoa formada por efluentes da bacia do Rio do Cobre) e criadouros de planorbídeos (Biomphilaria glabrata) - confirmados pelo Setor de Parasitologia do Laboratório Central do Estado (LACEN) da Secretaria de Saúde do Estado (LACEN, BA: comunicação pessoal, 2003). Também, médicos e enfermeiros da região do Subúrbio Ferroviário relatavam casos clínicos de esquistossomose, em crianças e adultos eliminando ovos de S. mansoni. Em vista disso, este estudo foi planejado com 0 objetivo de verificar a prevalência de esquistossomose na população escolar do bairro de São Bartolomeu, situado naquela região da Cidade do Salvador (Bahia) .

\section{MATERIAL E MÉTODOS}

Características da área de estudo. Ao longo dos séculos XVIII e XIX, o bairro de São Bartolomeu abrigou nas suas imediações diversos quilombos que reuniam centenas de negros rebelados contra 0 sistema escravista ${ }^{13}$; e como nesse bairro há mata atlântica e cachoeira, de onde se origina 0 riacho que corta toda a área, ainda hoje é utilizado como local de diversas cerimônias das religiões afro-brasileiras ${ }^{13}$. Por sua vez, os seus moradores utilizam essas fontes naturais de água como local de lazer (e.g., pesca e folguedos infantis) e para 0 cultivo de hortaliças.

Nesse riacho do Rio Cobre, nos seus efluentes e nas áreas alagadas (lagoas), há baixa vazão por minuto e foram identificados criadouros naturais de planorbídeos da espécie B. glabrata ( LACEN, BA: comunicação pessoal, 2003) .

0 bairro de São Bartolomeu faz parte do $11^{0}$ Distrito Sanitário de Salvador (BA), e contava em 2004 com população de 3.247 habitantes, sendo 530 (16,3\%) escolares na faixa etária de 7 a 14 anos $^{19}$. De acordo com o Programa de Saúde da Família (PSF), do Ministério da Saúde ${ }^{15}$, essa população foi distribuída em seis microáreas, conforme 0 local de residência, estando localizados na de número 4 os principais cursos naturais de água (Figura 1).

Na população do bairro de São Bartolomeu, a média da renda mensal não ultrapassa 2 salários-mínimos. No levantamento do Sistema de Informação de Atenção Básica (SIAB) ${ }^{19}$, realizado em 2003, foram cadastradas 879 famílias. Nos domicílios dessas famílias, a maioria ( $95,1 \%$ ) contava com abastecimento de água da rede pública, mas 707 (80,4\%) não estavam ligados ao sistema de esgotamento sanitário, sendo os dejetos lançados em valas afluentes dos cursos naturais de água.

População de estudo e exame parasitológico de fezes. Neste estudo de prevalência, realizado nos meses de janeiro a março de 2004, foram inicialmente selecionadas as 490 crianças, de 7 a 14 anos de idade, com matrícula regular nas escolas do bairro de São Bartolomeu.

0 exame parasitológico de fezes, pelo método de Kato-Katz ${ }^{11}$, foi realizado no Laboratório da 1 a Diretoria Regional de Saúde de Saúde (Salvador, Bahia); e os resultados foram fornecidos com 0 registro do número de ovos (por grama de fezes) de S. mansoni e se havia, ou não, a presença de ovos, cistos ou larvas de outros parasitas intestinais. Neste estudo, as crianças com 500 ou mais ovos do $S$. mansoni por grama de fezes foram consideradas como tendo elevada carga parasitária.

Inquérito epidemiológico, exame clínico e tratamento. Logo após a conclusão do inquérito coprológico, o responsável legal pela criança foi chamado à Unidade de Saúde da Família para responder ao questionário epidemiológico, sobre: dados demográficos ( sexo; idade; micoárea onde reside no bairro de São Bartolomeu; naturalidade; procedência; e residências anteriores, temporárias ou permanentes); histórico de viagens ou passeios para áreas de transmissão da esquistossomose; condições da moradia ( abastecimento de água; destino dos 


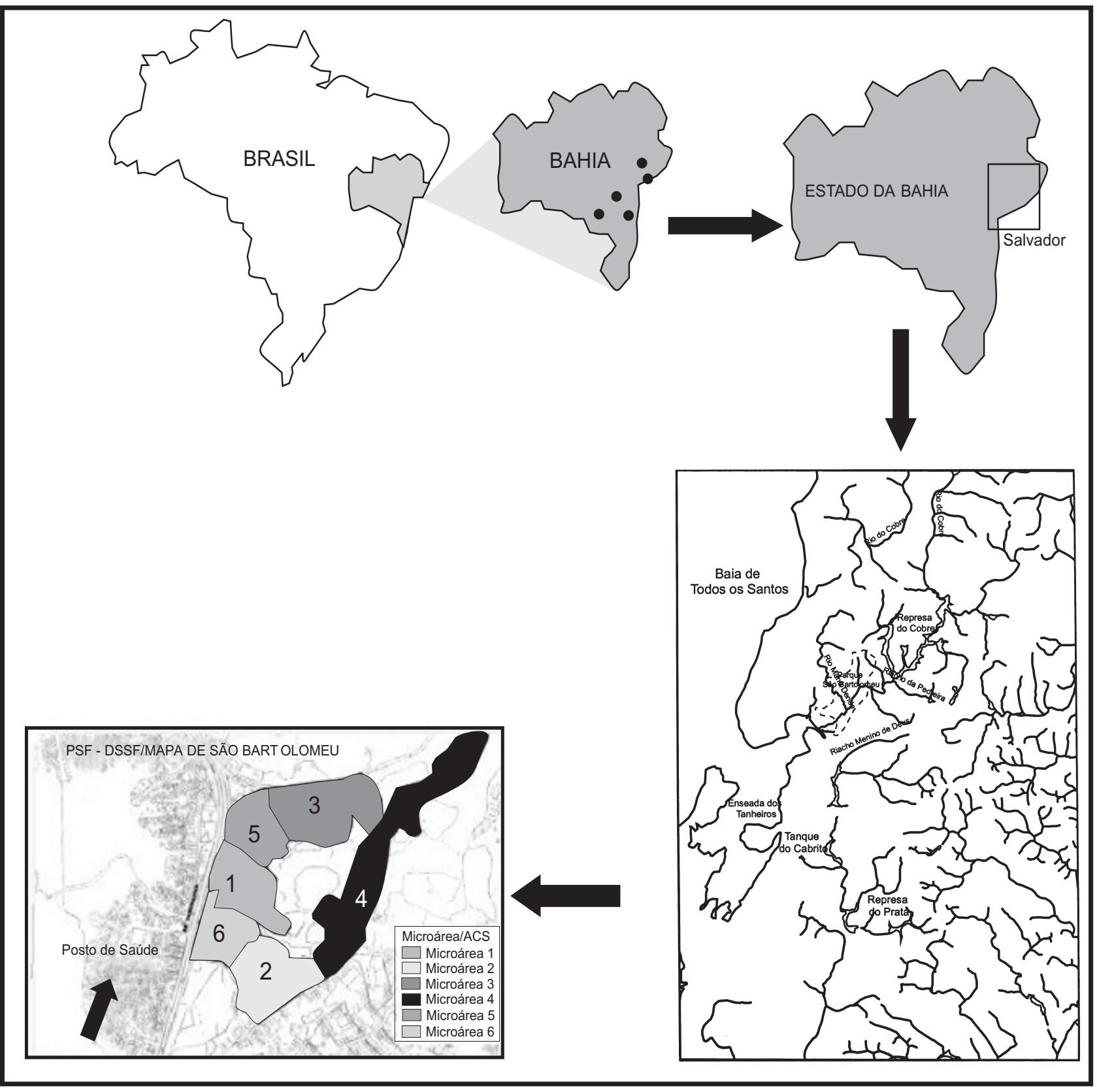

Figura 1 - Localização da área do estudo na Cidade do Salvador, Estado da Bahia. Brasil.

dejetos, tipo de construção e se havia vaso sanitário) ; e história dos contatos com as fontes naturais de água. Por ocasião desse levantamento, 0 entrevistador (ICSG) desconhecia o resultado do exame Kato-Katz, bem como o responsável (JTN) pelo exame clínico do abdômen das crianças.

No exame do abdômen, se palpáveis o fígado, o baço ou ambos eram anotadas suas dimensões e características, observando os parâmetros estabelecidos por Prata ${ }^{18}$ para a classificação das formas crônicas da esquistossomose. A criança teve 0 diagnóstico da forma clínica hepatosplênica (HE) se apresentasse baço palpável sem manobras respiratórias, e desde que no rebordo costal esquerdo ou além, e tendo hepatomegalia com lobo hepático esquerdo proeminente em relação ao direito; nesses casos, também foram anotadas a consistência hepática (se endurecida ou não) e as características da superfície do fígado (se lisa ou nodular). A forma clínica hepatointestinal (HI), foi distribuída em leve (HI-L) ou avançada (HI-A); nos pacientes com HI-L, o baço é impalpável e o fígado tem dimensões aumentadas, sem proeminência do lobo esquerdo, de consistência amolecida e com superfície lisa. Foram classificados como portadores da HI-A, se o baço era palpável à inspiração profunda abaixo do gradil costal esquerdo e 0 fígado, além de aumentado, tinha lobo esquerdo proeminente.

Após o exame clínico do abdômen, e em outro ambiente da Unidade de Saúde da Família, todos os casos com ovos de $S$. mansoni foram tratados com oxamniquine $(15 \mathrm{mg} / \mathrm{kg}$ de peso, dose única) e/ou receberam a prescrição com 0 tratamento de outras parasitoses intestinais. 
Análise estatística. Para análise estatística dos dados foi utilizando o Statistical Package for Social Sciences ( SPSS, versão 9.0). Conforme a indicação, nas variáveis qualitativas foi aplicado 0 teste do qui-quadrado $\left(\chi^{2}\right)$ e nas quantitativas, com distribuição normal, 0 teste tde Student. Nessas análises, a probabilidade do erro tipo I $<5 \%(\mathrm{p}<0,05)$ foi considerado significante.

\section{RESULTADOS}

Dos 490 selecionados, 268 (57\%) realizaram o exame parasitológico de fezes e em 81 (30,2\%) foram identificados ovos de $S$. mansoni, sendo $59(72,8 \%)$ de meninos e $22(27,2 \%)$ de meninas, e essa diferença foi estatisticamente significante (Tabela 1). A média de idade da população estudada foi de 10,8 $( \pm 2,4)$ anos, sendo estatisticamente maior $(\mathrm{p}<0,001)$ a dos eliminadores de ovos do S. mansoni $(11,8 \pm$ 2,3 anos) em comparação a dos negativos (10,4 $\pm 2,3$ anos) .

Tabela 1 - Freqüência de eliminadores de ovos de Schistosoma mansoni em escolares do bairro de São Bartolomeu (Salvador - Bahia), distribuídos por sexo e faixa etária.

\begin{tabular}{llccc}
\hline & & \multicolumn{3}{c}{ Ovos de Schistosoma mansoni } \\
\cline { 3 - 5 } Faixa etária (anos) & Sexo* & $\mathrm{n}^{0}$ & + & $\%$ \\
\hline $7 \longmapsto 10$ & a. feminino & 75 & 6 & 8,0 \\
& b. masculino & 65 & 19 & 29,2 \\
\hline $11 \longmapsto 14$ & c. feminino & 66 & 16 & 24,2 \\
& d. masculino & 62 & 40 & 64,5 \\
\hline Total & e. feminino & 141 & 22 & 15,6 \\
& f. masculino & 127 & 59 & 46,4
\end{tabular}

(*) "a" vs "b": $\chi^{2}=10,70 \mathrm{p}<0,002 ;$ "c" vs " $d ": \chi^{2}=21,07 \mathrm{p}<0,000005 ;$ " $\mathrm{e}$ " vs "f": $\chi^{2}=30,16$ p<0,0000001; "a" vs "c": $\chi^{2}=7,03$ p<0,009; "b" vs " $d$ ": $\chi^{2}=15,88$ $\mathrm{p}<0,00007$.

Entre as 81 crianças com ovos de $S$. mansoni, o número de ovos por grama de fezes variou de 24 a 2.160 ovos/g de fezes, sendo carga parasitária elevada observada em $25(9,3 \%)$ das crianças avaliadas.

Na Tabela 2, foram assinaladas as freqüências de crianças com esquistossomose por microárea da região de estudo, sendo estatisticamente maior (53,3\%) a frequêencia observada nas crianças residentes na microárea 4 , seguida naquelas das microáreas 3 e 1, respectivamente, 33,3\% e 30\%. Porém, nas

Tabela 2 - Distribuição de portadores de ovos de Schistosoma mansoni, por microárea de estudo do bairro de São Bartolomeu (Salvador, BA, 2003).

\begin{tabular}{lccc}
\hline & & \multicolumn{2}{c}{ Ovos de Schistosoma mansoni } \\
\cline { 3 - 4 } Microárea* & Exame Kato-Katz $\left(\mathrm{n}^{0}\right)$ & + & $\%$ \\
\hline 1 & 20 & 12 & 30,0 \\
2 & 48 & 18 & 25,0 \\
3 & 52 & 32 & 33,3 \\
4 & 61 & 11 & 53,3 \\
5 & 60 & 2 & 18,3 \\
6 & 27 & 81 & 7,4 \\
\hline Total & 268 & 100,0
\end{tabular}

(*) 1 vs $2: \chi^{2}=0,18 p>0,67 ;[1+2]$ vs $3: \chi^{2}=0,93 p>0,33 ;[1+2+3]$ vs $5: \chi^{2}=2,92$ $p>0,09 ;[1+2+3+5]$ vs $4: \chi^{2}=14,35 p<0,0002 ;[1+2+3+5]$ vs $6: \chi^{2}=4,55$ $\mathrm{p}<0,04$. crianças das microáreas 1, 2, 3 e 5 foram estatisticamente semelhantes (Tabela 2) as freqüências de casos com esquistossomose. Na microárea 6, houve a menor $(7,4 \%)$ freqüência de crianças com esquistossomose.

0 exame clínico do abdômen foi realizado em 248 ( 90,9\%) crianças incluídas no estudo, e entre essas $16(6,4 \%)$ tinham a forma hepatointestinal avançada (HI-A) e outras $3(1,2 \%)$ com a forma clínica hepatoesplênica (HE). Todas essas 19 crianças com as formas clínicas crônicas mais avançadas da esquistossomose (16 HI-A; $3 \mathrm{HE}$ ) tinham carga parasitária elevada; e os 3 pacientes com a forma HE residiam na microárea 4 e são da mesma família. Também, essas 19 crianças (16 HI-A; $3 \mathrm{HE}$ ) nasceram e sempre residiram no bairro São Bartolomeu e os seus familiares ou responsáveis negaram residência, permanente ou temporária, ou viagens para outras localidades do interior do Estado da Bahia ou do Nordeste do Brasil. Os responsáveis pelas outras 62 crianças com ovos de S. mansoni também negaram viagens ou residência, temporária ou permanente, para outras localidades; e desse grupo, 53 foram examinadas e todas tinham a forma clínica HI-L.

Nas outras 176 crianças examinadas, e sem ovos de S. mansoni nas fezes, só duas houve relato de viagens ou residências para outras localidades do Estado da Bahia. Nesse grupo, a quase totalidade ( $\mathrm{n}=168$ ) apresentava exame clínico normal do abdômen e 8 tinham características da forma clínica HI-L. Essas últimas, e as 81 crianças com ovos de $S$. mansoni nas fezes, foram tratadas com oxamniquine, erecomendado ao médico do PSF-São Bartolomeu repetir 3 vezes 0 exame Kato-Katzapós 45 dias do tratamento, além do acompanhamento clínico regular dos 19 pacientes com as formas crônicas mais avançadas ( $\mathrm{HI}-\mathrm{Ae} \mathrm{HE}$ ) da esquistossomose.

Todos os meninos maiores de 10 anos relataram 2 ou mais contatos com as fontes naturais de água do bairro de São Bartolomeu, mas 1 ou mais contatos só foram referidos por $29(44,6 \%)$ dos meninos com 7 a 10 anos de idade. Também, entre as meninas, as de 7 a 10 anos de idade referiram menos contatos $(\mathrm{n}=5 ; 6,7 \%)$ do que aquelas com 11 a 14 anos $(\mathrm{n}=12 ; 18,2 \%)$.

\section{DISCUSSÃ0}

No último inquérito coproscópico, realizado pela FUNASA entre os anos de 1993 e 1994, a Cidade do Salvador foi considerada como área de baixa endemicidade para a esquitossomose ${ }^{8}$. Posteriormente, Prado e cols ${ }^{17}$ encontraram 2,2\% de prevalência da esquistossomose ao examinarem 1.131 amostras fecais de crianças de 7 a 14 anos residentes em vários bairros da Cidade do Salvador, mas a prevalência observada (30,2\%) na população infantil dessa mesma faixa etária, do bairro de São Bartolomeu, também com a realização de apenas um exame parasitológico de fezes, e a significativa frequêencia $(9,3 \%)$ de crianças com elevada carga parasitária de $S$. mansoni, indicam a presença de foco de transmissão da infecção nesse bairro.

Fatores relacionados à história da formação do bairro de São Bartolomeu ${ }^{13}$, associados as suas características 
socioeconômicas e ecológicas ${ }^{19}$, podem ser considerados como determinantes daquela prevalência e do encontro de crianças expostas às formas clínicas mais graves da esquistossomose mansônica. Não obstante, outros bairros periféricos de Salvador têm características semelhantes e isso justifica futuro estudo populacional mais abrangente, apesar dos resultados descritos por Prado e cols ${ }^{17}$.

A constatação na área estudada de todos os elementos do ciclo biológico do $S$. mansoni permite supor ser mesmo 0 bairro de São Bartolomeu, em Salvador (Bahia), uma área de transmissão com impacto sobre a saúde da população local. Também, a observação de crianças com elevada carga parasitária e algumas delas com formas clínicas crônicas e graves da esquistossomose, todas sem história de residências em outras localidades, não só reforça que essa área da Cidade do Salvador tem um foco de transmissão do $S$. mansoni como também recomenda medidas de controle por parte dos serviços públicos.

Adistribuição observada por sexo e faixa etária das crianças com ovos de $S$. mansoni sugere a influência de variáveis comportamentais. A maior frequêencia de eliminadores de ovos de $S$. mansoni nas crianças do sexo masculino decorre, muito provavelmente, porque os meninos são mais expostos ao ambiente peridomiciliar durante as atividades de lazer ${ }^{17}$. Isso de certo modo é reforçado pela constatação da maior freqüência de crianças com ovos do $S$. mansoni ser observada nos maiores de 10 anos de idade, quando a população infantil da região de São Bartolomeu é mais exposta ao ambiente peridoméstico.

A microárea 4 da área de estudo é um bom exemplo dessa situação, porque é entre as 6 microáreas a mais próxima das coleções hídricas naturais. Coerentemente, na microárea 4 foi 2,2 vezes maior a freqüência ( $53,3 \%$ vs $23,7 \%$ ) de escolares eliminando ovos de $S$. mansoni e entre as suas 61 crianças foram encontrados os três únicos casos com a forma clínica hepatosplênica da esquistossomose.

Por sua vez, a boa articulação da equipe deste estudo e a do PSF possibilitou o conhecimento mais abrangente sobre aquela comunidade inclusive do seu processo de formação históricosocial e da hipótese da provável introdução de $S$. mansoni na área pela comunidade de afro-descendentes residentes em quilombos formados no primeiro quartel do século XVIII ${ }^{13}$. Também, sem a capacidade de mobilização da comunidade pela equipe local do PSF, e facilidade de acesso as microáreas com reconhecida periculosidade pela violência urbana, tornaria quase inexequível a realização deste estudo ou seria causa de ainda maior número de crianças excluídas.

Na perspectiva do planejamento e da execução de programa de controle da esquistossomose, no bairro de São Bartolomeu e arredores, a efetiva participação da população local e dos profissionais da rede básica de saúde, bem como 0 desenvolvimento de estratégias aplicáveis àquela região da cidade do Salvador, são etapas fundamentais para lograr boa efetividade das ações e do controle duradouro ${ }^{478}$.

Em conclusão, o bairro de São Bartolomeu na Cidade do Salvador (BA) é uma importante reserva ecológica de mata atlântica e potencial local de turismo e lazer, mas a provável transmissão do S. mansoni limita sua exploração socioeconômica. Portanto, as medidas de controle nessa localidade, especialmente aquelas voltadas ao saneamento básico, também irão servir para a mais rápida revitalização do Parque Ecológico de São Bartolomeu e trazer melhorias na qualidade de vida da sua população.

\section{REFERÊNCIAS BIBLIOGRÁFICAS}

1. Amaral RS, Porto MAS. Evolução e situação atual do controle da esquistossomose no Brasil. Revista da Sociedade Brasileira de Medicina Tropical 27 ( supl III) : 73-90 1994.

2. Andrade ZA. A esquistossomose no Brasil após quase um século de pesquisas. Revista Sociedade Brasileira Medicina Tropical 35: 509-513, 2002.

3. Barbosa CS, Pieri OS, Solva CB, Barbosa FS. Ecoepidemiologia da esquistossomose urbana na ilha de Itamaracá, Estado de Pernambuco. Revista Saúde Pública ( São Paulo) 34:337-341, 2000.

4. Barbosa CS, Solva CB, Barbosa FS. Esquistossomose: reprodução e expansão da endemia no Estado de Pernambuco no Brasil. Revista Saúde Pública ( São Paulo) 6: 609-619, 1996.

5. Barbosa FS. Determination and control of schistosomiasis. Memórias do Instituto Oswaldo Cruz 90: 155-159, 1995.

6. Barreto AC. Esquistossomose mansônica na cidade de Salvador. Tese de Livre-Docência, Universidade Federal da Bahia, Salvador, 1960.

7. Barreto ML, Carmo EH. Esquistossomose mansônica no Estado da Bahia, Brasil: tendências históricas e medidas de controle. Cadernos de Saúde Pública 10: 425-439, 1994.

8. Barros FR. A produção e a ocorrência da esquistossomose mansônica no Distrito Sanitário Cabula/Beiru e as estratégias de vigilância para 0 nível local. Dissertação de Mestrado, Instituto de Saúde Coletiva/Universidade Federal da Bahia, Salvador, 1988.

9. Coura-Filho P. 1. Distribuição da esquistossomose no espaço urbano. 2. Aproximação teórica sobre a acumulação, concentração, centralização do capital e a produção de doenças. Cadernos de Saúde Pública 13: 415-424, 1997.

10. Freitas CA. Situação atual da esquistossomose no Brasil. Revista Brasileira de Malariologia e Doenças Tropicais 24: 3-63, 1972.

11. Katz N, Chaves A, Pellegrino J. A simple device for qualitative stool thicksemear trchnique in schistosomiasis mansoni. Revista Instituto Tropical São Paulo 14: 39-402, 1972.

12. Katz N, Guimarães CT, Souza CP, Santos OC. Sobre um foco urbano de esquistossomose em área metropolitana da região sudeste do Brasil. Revista Saúde Pública ( São Paulo) 27: 210-213, 1993.

13. Machado GB. Urbanização e meio ambiente: 0 Parque de São Bartolomeu no Subúrbio Ferroviário de Salvador. Monografia, Faculdade de Ciências Econômicas/Universidade Federal da Bahia, Salvador, 1995.

14. Martin JE, Amorim A, Schall VT. Acute schistosomiasis outbreak in the metropolitan area of Belo Horizonte, Minas Gerais: alert about the risk of unnoticed transmission increased by growing rural tourism. Memórias do Instituto Oswaldo Cruz 98:745-750, 2003.

15. Ministério da Saúde. Brasil. Programa de Saúde da Família. Disponível em http://www.saude.gov.br, recuperado on line em 16.08.2004, 2004.

16. Pellon $A B$, Teixeira I. Distribuição geográfica da esquistossomose mansônica no Brasil. Ministério da Educação e Saúde, Rio de Janeiro, 1950.

17. Prado MS, Barreto ML, Strina A, Faria AJS, Nobre AA, Jesus SR. Prevalência e intensidade da infecção por parasitas intestinais em crianças na idade escolar na Cidade de Salvador ( Bahia, Brasil) . Revista Sociedade Brasileira Medicina Tropical 34: 99-101, 2001.

18. Prata A. Como caracterizar a forma hepatoesplênica da esquistossomose. In: Prata A, Aboin E (eds) II Simpósio sobre Esquistossomose. Diretoria de Saúde da Marinha/Universidade Federal da Bahia, Salvador, p. 175-184, 1970.

19. Sistema de Informação da Atenção Básica (SIAB). Distrito Sanitário Subúrbio Ferroviário. Prefeitura Municipal de Salvador/Secretaria Municipal de Saúde, Salvador, 2004. 